\title{
Numerical study on the effect of waterway shape on ship wave characteristics
}

\author{
by \\ Chun Beom Hong*, Student Member \\ Hidemi Mutsuda*, Member \\ Yasuaki Doi *, Member
}

\begin{abstract}
Summary
Ship wave characteristics in a shallow and restricted waterway whose depth and breadth change in longitudinal direction are studied numerically.

The Euler and continuity equations are employed for the present study. Boundary fitted and moving grid system is adopted in the numerical simulation. The convection term in the governing equations and the free surface boundary condition are solved by Cubic Interpolated Pseudo particle (CIP) algorithm in order to get high resolution of wave height in far field as well as near field.

The present numerical scheme is applied to predict ship waves in shallow and restricted waterway whose depth and breadth vary in longitudinal direction.
\end{abstract}

\section{Introduction}

Contrary to ship design concept in the past, the environmental impact by ship waves, such as risk for people on beach, erosion of coast and unacceptable ship movement in harbors, becomes significant design criteria in the present time. Danish Hydraulic Institute and Maritime Authority ${ }^{1)}$ reported the environmental impact in Arhus-Kalundborg route and Xontech Inc. also introduced the environmental problem caused by ship waves in Rich Passage ${ }^{2)}$. Stumbo et al. ${ }^{3)}$ and Kirkegaatd et al. ${ }^{4}$ carried out full scale measurement and prediction of ship wave amplitude by panel code and then tried to establish criteria of no harmful ship waves.

In the study of ship wave phenomena in restricted waterway, Ertekin $^{5)}$ conducted model test to investigate the relation between blockage coefficient and characteristics of solitons and Chen et al. ${ }^{\text {) }}$ simulated the wave phenomena in restricted waterway with constant depth and breadth.

In general, water depth and breadth in real ship route are not constant and the map around Rich passage in U.S.A is shown in Fig. 1 as an example. The dotted line indicates a ferry route. In order to predict ship waves, which is more similar to wave phenomena in real situation, it is necessary to investigate ship wave phenomena in shallow and restricted waterway whose depth and breadth vary in longitudinal direction. Madsen et al. ${ }^{7}$ ) have studied phenomena of wave propagation on slowly varying bathymetry from deep to shallow water and showed special wave phenomena.

In this paper, the fundamental study of ship wave phenomena in restricted waterway whose depth and breadth vary in longitudinal direction is performed numerically. In order to obtain an accurate solution without expensive computational effort, CIP method ${ }^{8)}$ is adopted. The numerical scheme is validated by comparison of numerical results with experimental one in deep water case. The numerical method is applied to predict ship wave characteristics when a ship passes the restricted waterway whose depth and breadth vary in longitudinal direction.

* Graduate School of Engineering,

Hiroshima University

Received 10th July 2002

Read at the Autumn meeting 14, 15th Nov. 2002

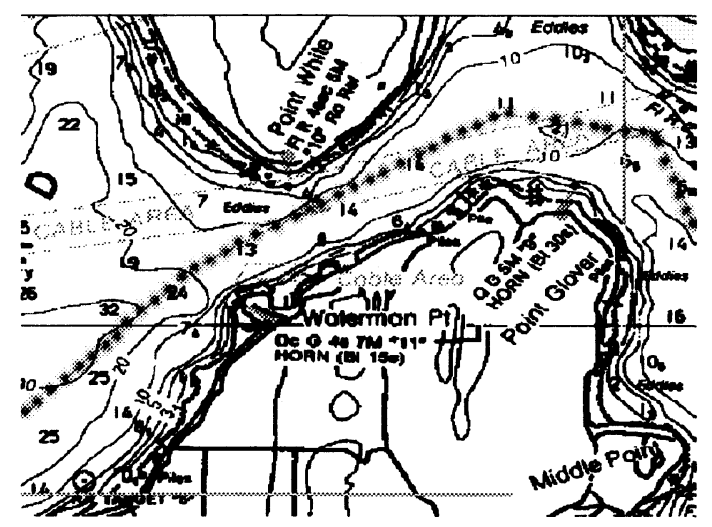

Fig. 1 Sea map around Rich Passage

\section{Waterway model}

The configuration of restricted waterway investigated in the present study is shown in Fig. 2. The depth of waterway varies in longitudinal direction. The water depth equals to $0.2 \mathrm{~L}$ (L : ship length) where a ship starts and the depth varies from $0.2 \mathrm{~L}$ to $0.1 \mathrm{~L}$. Froude number based on the ship length $(\mathrm{Fn})$ is 0.4025 , while Froude number based on the water depth (Fnh) is 0.90 where water depth equals to $0.2 \mathrm{~L}$. After the ship transits the step, depth Froude number varies from 0.9 to 1.27 which corresponds to super-critical condition.

\section{Governing equations}

Three-dimensional incompressible Euler and continuity equations including the effect of grid movement are employed for the present numerical simulation of ship waves. The dimensionless form of the governing equations is expressed as follows, 


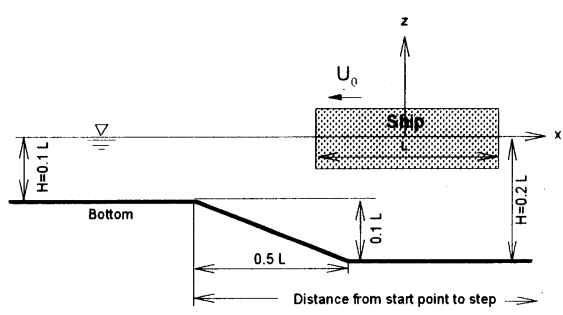

Fig. 2 Side view of restricted waterway

$$
\begin{aligned}
& \frac{\partial u}{\partial \tau}+\left(u-u_{g}\right) \frac{\partial u}{\partial x}+\left(v-v_{g}\right) \frac{\partial u}{\partial y}+\left(w-w_{g}\right) \frac{\partial u}{\partial z}=-\frac{\partial p}{\partial x} \\
& \frac{\partial v}{\partial \tau}+\left(u-u_{g}\right) \frac{\partial v}{\partial x}+\left(v-v_{g}\right) \frac{\partial v}{\partial y}+\left(w-w_{g}\right) \frac{\partial v}{\partial z}=-\frac{\partial p}{\partial y} \\
& \frac{\partial w}{\partial \tau}+\left(u-u_{g}\right) \frac{\partial w}{\partial x}+\left(v-v_{g}\right) \frac{\partial w}{\partial y}+\left(w-w_{g}\right) \frac{\partial w}{\partial z}=-\frac{\partial p}{\partial z} \\
& \nabla \cdot \vec{v}=0
\end{aligned}
$$

where $p=p^{*}+\frac{z}{F n^{2}}, p^{*}=p^{* *}-p_{a i r}$ and $F n=\frac{U_{0}}{\sqrt{g L}}$.

$x, y$ and $z$ are the Cartesian coordinates; $x$ in uniform flow, $y$ in lateral, $z$ in vertical direction respectively. The origin of the coordinate locates at the center of midship on the undisturbed free surface (Fig. 2) and is fixed on the ship. $\tau$ is the dimensionless time in fixed computational domain. $u, v$ and $w$ are the velocity components and $u_{g}, v_{g}$ and $w_{g}$ indicate velocity components of grid point in $x, y$ and $z$ directions. $\vec{v}$ denotes velocity vector. $p, p^{* *}, p_{\text {air }}$, $U_{0}, g$ and $L$ indicate modified pressure, pressure, atmospheric pressure, uniform flow velocity, gravity acceleration and the ship length. All variables in the governing equations are non-dimensionalized by ship length and uniform flow velocity.

\section{Boundary conditions}

Neglecting effects of surface tension and viscosity, the pressure acting on the free surface is set to be the atmospheric pressure as shown in equation (3). The velocity of fluid on the free surface must satisfy the kinematic boundary condition as shown in equation (4).

$$
\begin{aligned}
& p^{*}=p_{\text {air }} \\
& \frac{\partial \zeta}{\partial t}=w-u \frac{\partial \zeta}{\partial x}-v \frac{\partial \zeta}{\partial y}
\end{aligned}
$$

where $\zeta(\mathrm{x}, \mathrm{y}, \mathrm{t})$ is the wave height and $t$ is the dimensionless time in moving physical domain.

Free slip boundary condition is used for the wall boundary in Eulerian manner of description ${ }^{9}$. Because waterway depth and breadth vary in longitudinal direction, the velocity boundary condition on the wall is given as follows,

$$
\begin{aligned}
& \vec{v}_{E}=\vec{v}-U_{0} \\
& \vec{v}_{E} \cdot \vec{n}=0 \\
& \frac{\partial V_{t}}{\partial n}=0
\end{aligned}
$$

where, $\vec{v}_{E}$ and $V_{t}$ are velocity vector and the tangential velocity components in Eulerian coordinate system respectively. The unit vector normal to the wall is denoted by $\vec{n}$. The pressure boundary condition on the body surface is derived to satisfy the momentum equation.

On the upstream boundary of the computational domain, uniform flow condition is applied, while zero gradient condition is given on the outer boundary. On the center plane, the symmetric boundary condition is applied.

\section{Numerical scheme}

\subsection{CIP method}

In the present study, CIP method is employed for three dimensional ship waves simulation in curvilinear coordinate system. Discretized form of equation (1) is split into two stages, non-advection and advection stage, assuming a small time increment.

In the non-advection stage, $\vec{v}^{*}$ and $\zeta^{*}$ are calculated by equations (8) and (9).

$$
\begin{aligned}
& \frac{\vec{v}^{*}-\vec{v}^{m}}{\Delta t}=-\nabla p^{m} \\
& \frac{\zeta^{*}-\zeta^{m}}{\Delta t}=w^{m}
\end{aligned}
$$

where superscripts ${ }^{*}$ and $m$ denote time step in nonadvection stage and $\Delta t$ means time increment. Taking the divergence of equation (8), Poisson equation for pressure can be derived.

$$
\nabla^{2} p^{*}=-\frac{D^{m}}{\Delta t}
$$

where $D$ represents the divergence of velocity vector. Equations (8), (9) and (10) are transformed into a body fitted coordinate system and discretized by finite difference method with the second order accuracy.

After $\vec{v}^{*}$ and $p^{*}$ are obtained in non-advection stage, CIP method, which can express the behavior of the solution and its derivatives inside grid cell, is applied to solve the flow advection.

The function $(f)$, such as velocity component or wave elevation, and its derivatives $\left(\partial f / \partial \xi_{i}\right)$ are expressed by cubic polynomial in curvilinear coordinate system as shown in equations (11) (14). $\xi_{1}, \xi_{2}$ and $\xi_{3}$ are curvilinear coordinates and $U_{c}, V_{c}$ and $W_{c}$ denote contra variant velocity components in $\xi_{1}, \xi_{2}$ and $\xi_{3}$ directions. The coefficients of $c_{1} \sim c_{16}$ are determined by substitution of $f$ and its derivatives at the eight vertices of the cell. 
$f^{m+1}(i, j, k)$

$=\left(\left(c_{1} \Delta \xi_{1}+c_{4} \Delta \xi_{2}+c_{7} \Delta \xi_{3}+c_{11}\right) \Delta \xi_{1}+c_{14} \Delta \xi_{2}+\frac{\partial f^{*}}{\partial \xi_{1}}(i, j, k)\right) \Delta \xi_{1}$

$+\left(\left(c_{2} \Delta \xi_{2}+c_{5} \Delta \xi_{1}+c_{8} \Lambda \xi_{3}+c_{12}\right) \Delta \xi_{2}+c_{15} \Delta \xi_{3}+\frac{\partial f^{*}}{\partial \xi_{2}}(i, j, k)\right) \Delta \xi_{2}$

$+\left(\left(c_{3} \Delta \xi_{3}+c_{6} \Lambda \xi_{1}+c_{9} \Delta \xi_{2}+c_{13}\right) \Delta \xi_{3}+c_{16} \Delta \xi_{1}+\frac{\partial f^{*}}{\partial \xi_{3}}(i, j, k)\right) \Delta \xi_{3}$

$+c_{10} \Delta \xi_{1} \Delta \xi_{2} \Delta \xi_{3}+f^{*}(i, j, k)$

$\frac{\partial f^{m+1}}{\dot{\partial} \xi_{1}}(i, j, k)$

$=\left(3 \times c_{1} \Delta \xi_{1}+2\left(c_{4} \Delta \xi_{2}+c_{7} \Delta \xi_{3}+c_{11}\right)\right) \Delta \xi_{1}$

$+\left(c_{5} \Delta \xi_{2}+c_{10} \Delta \xi_{3}+c_{14}\right) \Delta \xi_{2}+\left(c_{6} \Delta \xi_{3}+c_{16}\right) \Delta \xi_{3}$

$+\frac{\partial f^{*}}{\partial \xi_{1}}(i, j, k)-\Delta t\left(\frac{\partial f^{*}}{\partial \xi_{1}} \frac{\partial U_{c}}{\partial \xi_{1}}+\frac{\partial f^{*}}{\partial \xi_{2}} \frac{\partial V_{c}}{\partial \xi_{1}}+\frac{\partial f^{*}}{\partial \xi_{3}} \frac{\partial W_{c}}{\partial \xi_{1}}\right)$

$\frac{\partial f^{m+1}}{\dot{d} \xi_{2}}(i, j, k)$

$=\left(3 \times c_{2} \Delta \xi_{2}+2\left(c_{5} \Delta \xi_{1}+c_{8} \Delta \xi_{3}+c_{12}\right)\right) \Delta \xi_{2}$

$+\left(c_{9} \Delta \xi_{3}+c_{10} \Delta \xi_{1}+c_{15}\right) \Delta \xi_{3}+\left(c_{4} \Delta \xi_{1}+c_{14}\right) \Delta \xi_{1}$

$+\frac{\partial f^{*}}{\partial \xi_{2}}(i, j, k)-\Delta t\left(\frac{\partial f^{*}}{\partial \xi_{1}} \frac{\partial U_{c}}{\partial \xi_{2}}+\frac{\partial f^{*}}{\partial \xi_{2}} \frac{\partial V_{c}}{\partial \xi_{2}}+\frac{\partial f^{*}}{\partial \xi_{3}} \frac{\partial W_{c}}{\partial \xi_{2}}\right)$

$\frac{\partial f^{m+1}}{\partial \xi_{3}}(i, j, k)$

$=\left(3 \times c_{3} \Delta \xi_{3}+2\left(c_{6} \Delta \xi_{1}+c_{9} \Delta \xi_{2}+c_{13}\right)\right) \Delta \xi_{3}$

$+\left(c_{7} \Delta \xi_{1}+c_{10} \Delta \xi_{2}+c_{16}\right) \Delta \xi_{1}+\left(c_{8} \Delta \xi_{2}+c_{15}\right) \Delta \xi_{2}$

$+\frac{\partial f^{*}}{\partial \xi_{3}}(i, j, k)-\Delta t\left(\frac{\partial f^{*}}{\partial \xi_{1}} \frac{\partial U_{c}}{\partial \xi_{3}}+\frac{\partial f^{*}}{\partial \xi_{2}} \frac{\partial V_{c}}{\partial \xi_{3}}+\frac{\partial f^{*}}{\partial \xi_{3}} \frac{\partial W_{c}}{\partial \xi_{3}}\right)$

$$
\begin{aligned}
\Delta \xi_{1} & =-U_{c} \Delta t \\
\Delta \xi_{2} & =-V_{c} \Delta t \\
\Delta \xi_{3} & =-W_{c} \Delta t
\end{aligned}
$$

where $U_{c}, V_{c}$ and $W_{c}$ are contravariant velocity components.

$$
\begin{aligned}
& U_{c}=\left(\xi_{1}\right)_{x}\left(u-u_{g}\right)+\left(\xi_{1}\right)_{y}\left(v-v_{g}\right)+\left(\xi_{1}\right)_{z}\left(w-w_{g}\right) \\
& V_{c}=\left(\xi_{2}\right)_{x}\left(u-u_{g}\right)+\left(\xi_{2}\right)_{y}\left(v-v_{g}\right)+\left(\xi_{2}\right)_{z}\left(w-w_{g}\right) \\
& W_{c}=\left(\xi_{3}\right)_{x}\left(u-u_{g}\right)+\left(\xi_{3}\right)_{y}\left(v-v_{g}\right)+\left(\xi_{3}\right)_{z}\left(w-w_{g}\right)
\end{aligned}
$$

\subsection{MAC method}

The present method is based on the method proposed Welch et al. ${ }^{10)}$. The Poisson equation for pressure includes the divergence of the convection term, which is different from the equation used in CIP method. The velocity components at new time step are extrapolated by Euler explicit scheme. The convection terms and the kinematic free surface boundary condition are discretized by the third order upwind scheme. All the other spatial derivative terms are discretized by the second order centered difference scheme.

\section{Pilot computations}

The ship waves calculated by CIP method in deep water are compared with those by MAC method and experimental ones ${ }^{11)}$. The comparison of wave profile for Wigley hull at $F n=0.289$ is shown in Fig. 3. The wave elevations on the hull computed by the two methods are similar to the measured ones, but wave patterns computed by the two methods are different from each other. The computed wave pattern by CIP method shows well propagation in far field while that by MAC method does not so much. The number of grids per one wave length in far field is 13 in both cases. Through the comparison of computations, it is found that CIP method has a merit to predict ship waves accurately in far field.

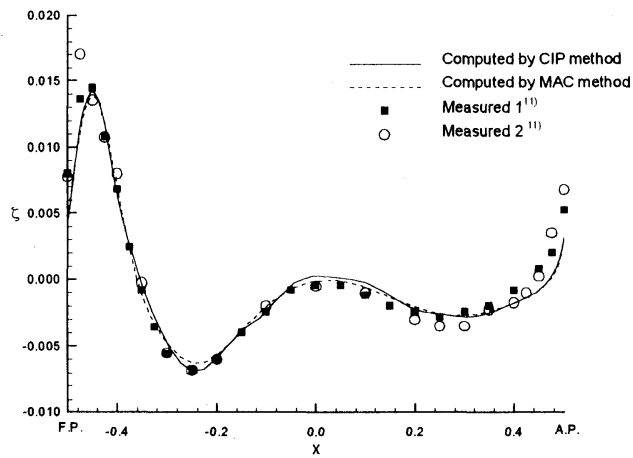

Fig. 3 Comparison of wave profiles on Wigley hull $(\mathrm{Fn}=0.289)$

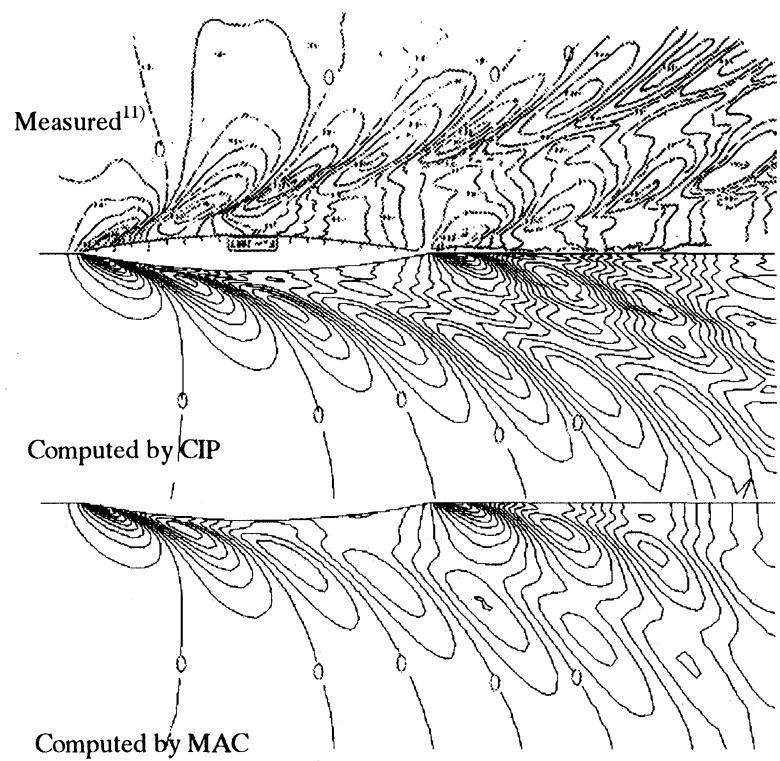

Fig. 4 Comparison of wave patterns between computed and measured $(\mathrm{Fn}=0.289$, Interval of contour is $0.00125 \mathrm{~L})$

Since ship wave phenomena in restricted waterway are unsteady at sub-critical and critical condition, the dependency of time increment is examined. The breadth of waterway is 
selected to be $0.5 \mathrm{~L}$ in the present computation, because such a narrow waterway intensifies the wave height.

The wave profiles on the hull surface and the center plane as time passes are shown in Figs. 5 and 6. The locations of F.P. and A.P. in $\mathrm{x}$ coordinate are -0.5 and 0.5 respectively. The difference of wave height due to the different time increment seems to be unsteadiness caused by the change of bottom shape. As the time increment becomes smaller, the difference between the computations grows less and less. If the time increment is less than or equals to 0.0005 , the solution does not depend on the time increment. Because of that point, 0.0005 is selected as a standard time increment.

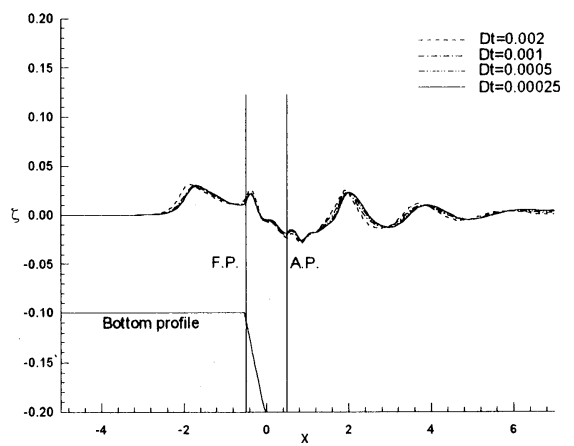

Fig. 5 Comparison of wave profiles for different time increment

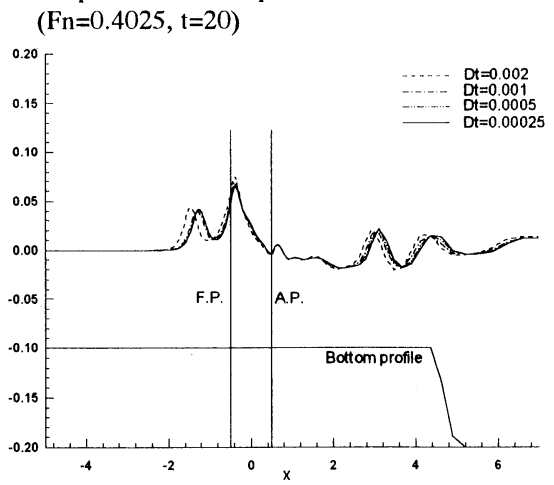

Fig. 6 Comparison of wave profiles for different time increment $(\mathrm{Fn}=0.4025, \mathrm{t}=25)$

In order to examine the dependency of grid size in longitudinal and lateral directions, computations with different grid size are carried out.

The grid configuration to examine the dependency of grid size in lateral direction is shown in Table. 1. Fig. 7 shows a part of grid system on water plane for case 3 . The wave elevations on the hull surface and the center plane for different grid resolution are shown in Figs.8 and 9. The wave elevations of Case 2 and Case 3 are much the same. Therefore, the grid system of Case 2 is selected as a standard grid in lateral direction.

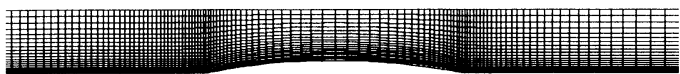

Fig. 7 Grid system on water plane for case 3
Table. 1 Grid configuration to examine grid dependency in lateral direction

\begin{tabular}{|c|c|c|c|}
\hline & No. of grid & $\begin{array}{c}\text { Minimum grid } \\
\text { size }\end{array}$ & $\begin{array}{c}\text { Maximum } \\
\text { grid size }\end{array}$ \\
\hline Case1 & 15 & 0.005 & 0.06 \\
\hline Case2 & 20 & 0.005 & 0.03 \\
\hline Case3 & 30 & 0.005 & 0.015 \\
\hline
\end{tabular}

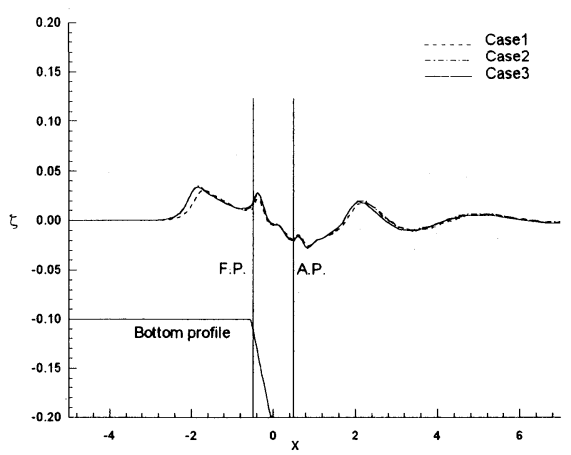

Fig. 8 Comparison of wave profiles for different grid in lateral direction $(\mathrm{Fn}=0.4025, \mathrm{t}=20)$

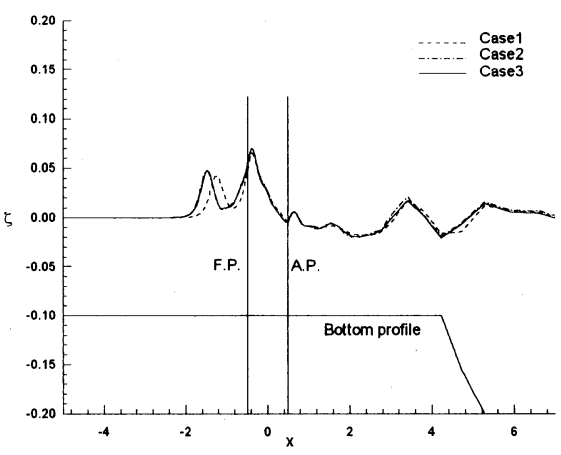

Fig. 9 Comparison of wave profiles for different grid in lateral direction $(\mathrm{Fn}=0.4025, \mathrm{t}=25)$

The calculated grid configuration in longitudinal direction is shown in Table. 2. The size of grid in the zone from one ship length to three times of ship length ahead of F.P. is constant. The computed wave elevations on the hull surface and the center plane are shown in Figs. 10 and 11. The difference of the wave elevation according to the grid sizes becomes small if the size is less than 0.04 (Case5). Therefore Case 5 is chosen as a standard grid in longitudinal direction.

Table. 2 Grid configuration to examine grid dependency in longitudinal direction.

\begin{tabular}{|c|c|c|c|}
\hline & $\begin{array}{c}\text { Size of grid } \\
\text { at F.P. }\end{array}$ & $\begin{array}{c}\text { Size of grid at one ship } \\
\text { length ahead of stem }\end{array}$ & $\begin{array}{c}\text { No. of } \\
\text { grids }\end{array}$ \\
\hline Case4 & 0.01 & 0.08 & 258 \\
\hline Case5 & 0.01 & 0.04 & 196 \\
\hline Case6 & 0.01 & 0.02 & 161 \\
\hline
\end{tabular}




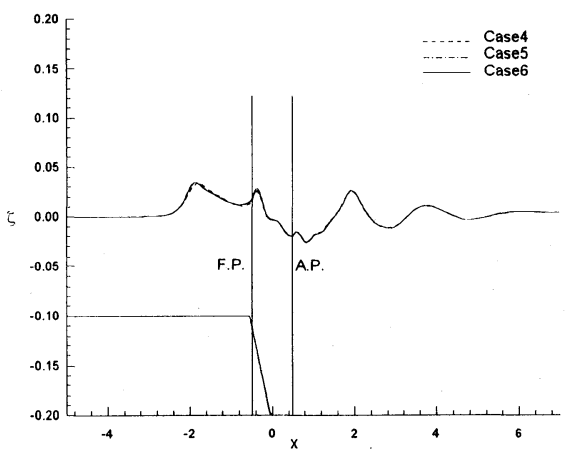

Fig. 10 Comparison of wave profiles for different grid in longitudinal direction $(\mathrm{Fn}=0.4025, \mathrm{t}=20)$

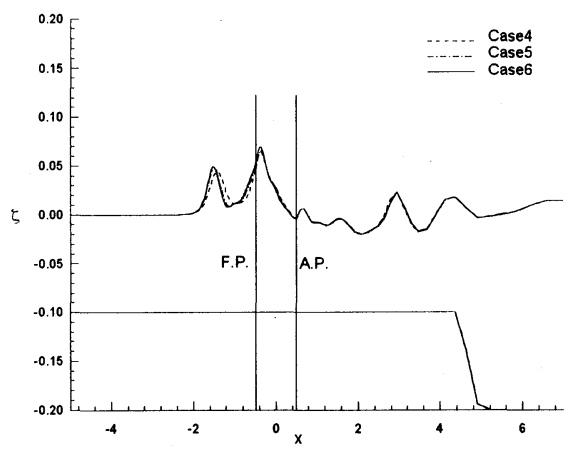

Fig. 11 Comparison of wave profiles for different grid in longitudinal direction $(\mathrm{Fn}=0.4025, \mathrm{t}=25)$

\section{Applications}

The numerical method is applied to predict waves for Wigley hull in the restricted waterway (Fig. 2). The simulations are performed in four types of waterway with different breadth (B). The breadth of waterway and distance from start point to the step on the bottom are shown in Table. 3. In order to generate a solitary wave for all cases before ship arrives at the step, different distances from the start point to the step are used for the computation. The different distance is caused by the period of solitary wave which is inversely proportional to waterway breadth. Froude number based on the ship length is 0.4025 and Froude number based on the water depth is 0.9 before the ship reaches the step. After the ship transits the step, depth Froude number becomes 1.272 .

The evolution of wave patterns for $\mathrm{B}=0.5 \mathrm{~L}$ is shown in Fig. 12. In the figure, 'step' indicates the location of the step on the bottom and $t_{s}$ indicates the dimensionless time since the ship transits the step. Bright region and dark region mean positive and negative wave height respectively. The solitary wave moves ahead of ship until $t_{s}=0.0$ while the direction of wave propagation turns reverse after that stage. The change of the direction of waves propagation is caused by the water depth because the wave velocity is proportional to the water depth. The solitary wave moves in the reverse direction and merged with the bow wave. After $t_{s}=10.0$, very large wave height is observed. After this stage, another solitary waves are generated as shown in Fig. 12. The generation of the second solitary wave seems to be the effect of waterway breadth.

Table. 3 Summary of distance from start point to step on the bottom.

\begin{tabular}{|c|c|}
\hline Breadth of waterway & Distance from start point to step \\
\hline $\mathrm{B}=0.5 \mathrm{~L}$ & $20 \mathrm{~L}$ \\
\hline $\mathrm{B}=1.0 \mathrm{~L}$ & $30 \mathrm{~L}$ \\
\hline $\mathrm{B}=1.5 \mathrm{~L}$ & $40 \mathrm{~L}$ \\
\hline $\mathrm{B}=$ infinite & $25 \mathrm{~L}$ \\
\hline
\end{tabular}

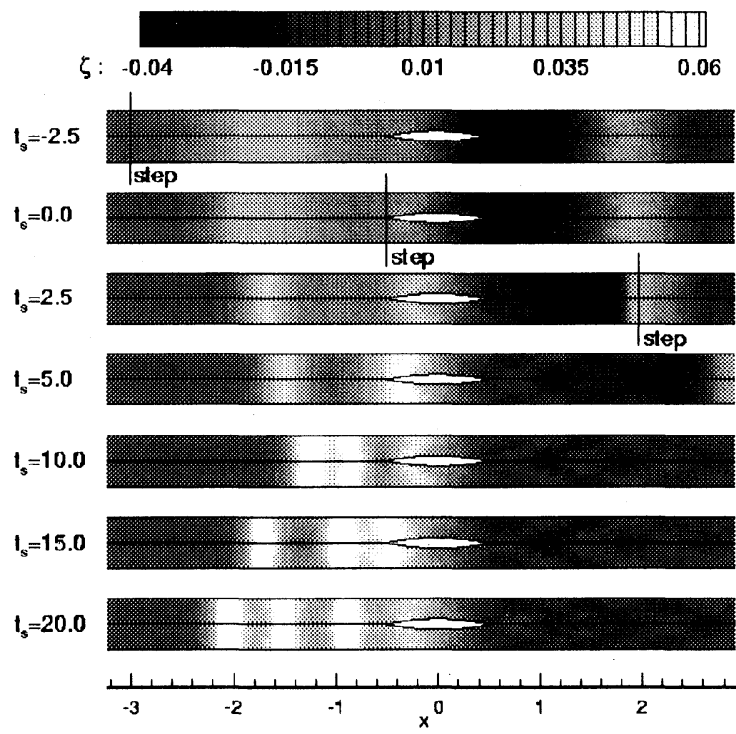

Fig. 12 Evolution of computed wave patterns ( $F n=0.4025$, $\mathrm{B}=0.5 \mathrm{~L})$

In the case of $B=1.0 \mathrm{~L}$, the second solitary wave is not observed as shown in Fig. 12, but a steep and stationary wave, which does not move forward or backward, is generated in front of the ship as shown in Fig. 13.

The stationary solitary wave observed in Fig. 13 does not appear in the case of $\mathrm{B}=1.5 \mathrm{~L}$ (Fig. 14). The disappearance of stationary solitary wave is caused by the increase of breadth in comparison with the previous one. After $t_{s}=-2.5$, the solitary wave moves in the reverse direction of ship movement, thus the wave height is intensified when the solitary wave and the bow wave are superimposed at $t_{s}=12.5$. The largest wave appears on the side wall at that moment and the superimposed wave moves to the rear of stern. As shown in Fig. 15, maximum wave height is observed when $t_{s}=12.5$, which indicates that the ship locates 12 times of the ship length ahead of the step. 


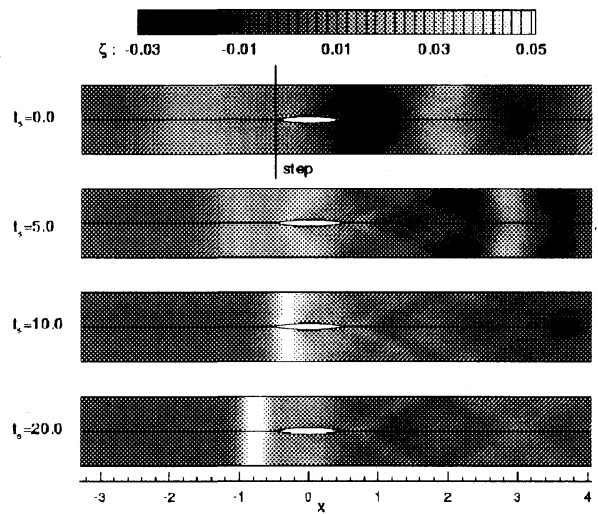

Fig. 13 Evolution of computed wave patterns $(F n=0.4025$, $\mathrm{B}=1.0 \mathrm{~L})$

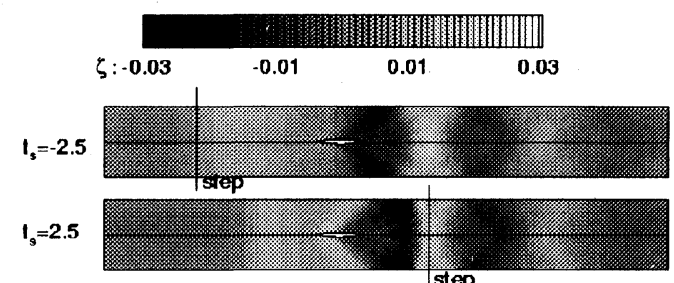

step
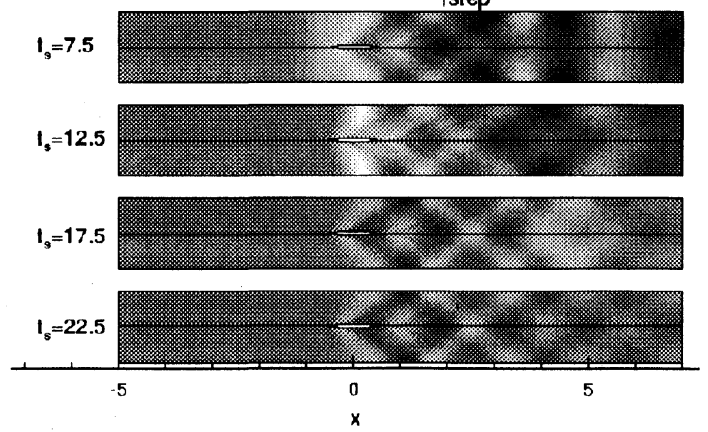

Fig. 14 Evolution of computed wave patterns $(F n=0.4025$, $\mathrm{B}=1.5 \mathrm{~L}$ )

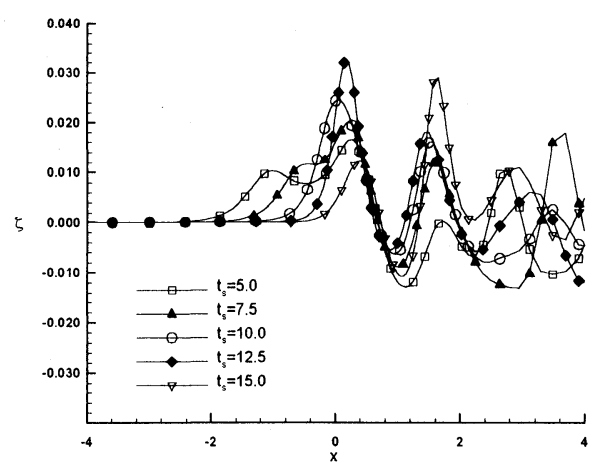

Fig. 15 Computed wave elevation on the side wall $(\mathrm{Fn}=0.4025$, $\mathrm{B}=1.5 \mathrm{~L})$
Finally the prediction of ship waves for shallow water without side wall is performed. Fig. 16 shows the evolution of wave patterns as time passes. After the ship transits the step, a developing bow wave crest is observed, while the wave observed around $\mathrm{x}=1.5 \mathrm{~L}$ and $\mathrm{y}=0.6 \mathrm{~L}$ at $t_{s}=2.5$ moves to the rear of stern and disappears at $t_{s}=10.0$.
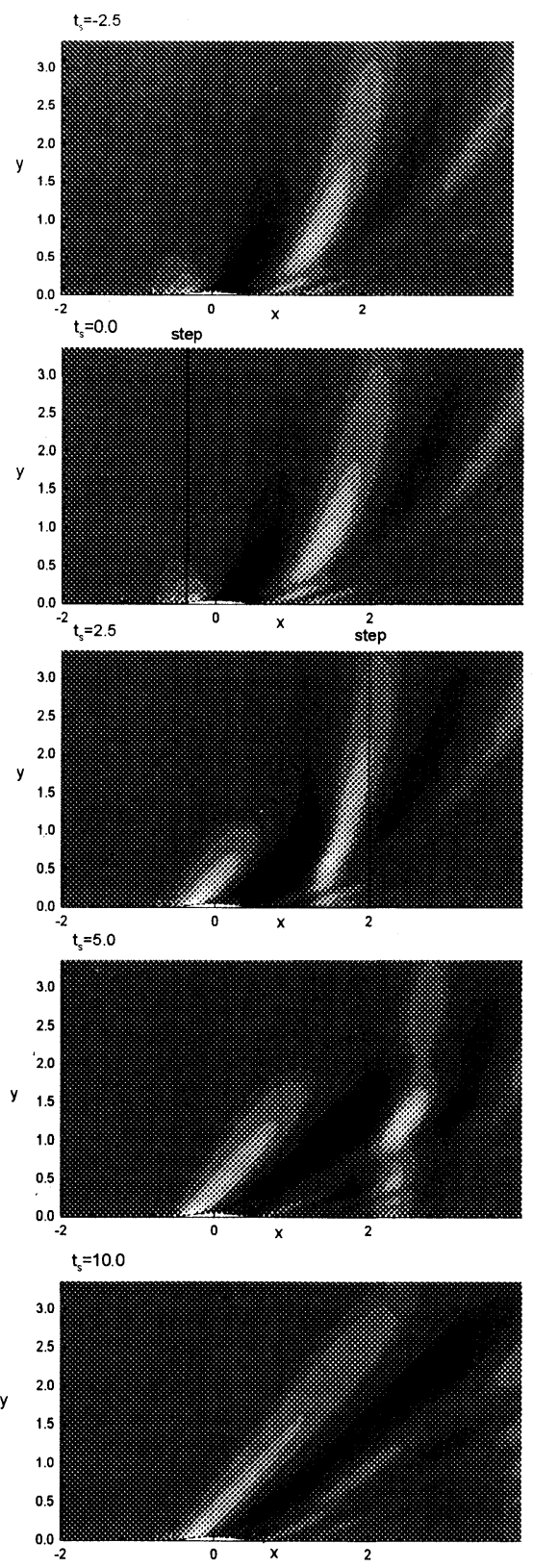

Fig. 16 Evolution of computed wave patterns $(F n=0.4025$, $\mathrm{B}=$ infinite) 
The effect of change of waterway breadth on ship wave in a restricted waterway is investigated. The distance from the start point to the step on side wall is $40 \mathrm{~L}$. The breadth is $1.5 \mathrm{~L}$ before $t_{s}=0.0$, while the breadth changes to $1.0 \mathrm{~L}$ after $t_{s}=0.0$. The depth of waterway is $0.2 \mathrm{~L}$ uniformly. Froude numbers based on the ship length and the water depth equal to 0.4025 and 0.9 respectively. The time evolution of wave patterns is shown in Fig. 17. The ship wave characteristics are different from the previous results for waterway whose depth vary in longitudinal direction. Unsteady effect due to the changes of breadth does not appear.

However the stationary solitary wave is observed in restricted waterway whose depth and breadth vary in longitudinal direction simultaneously as shown in Fig. 18. Through the computations, it is found that the dominant effect on the ship waves, such as appearance of solitary or stationary wave, is not the change of the water breadth but the water depth.

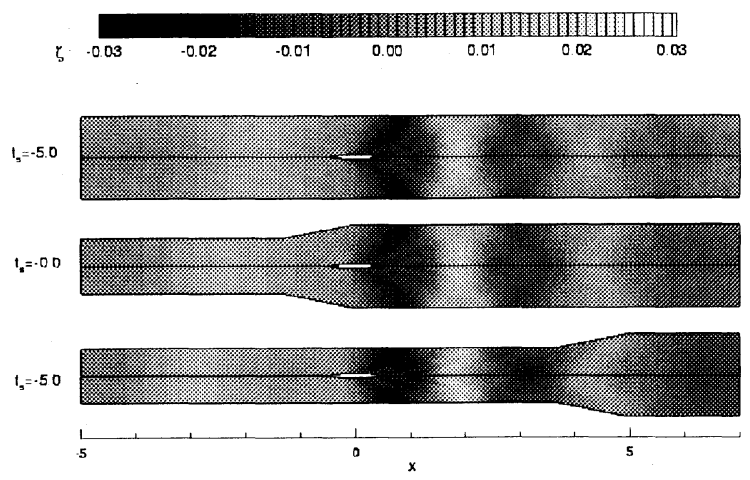

Fig. 17 Evolution of computed wave patterns $(\mathrm{F} n=0.4025$, $\mathrm{B}=1.5 \mathrm{~L} \sim 1.0 \mathrm{~L}, \mathrm{~h}=0.2 \mathrm{~L}$ )
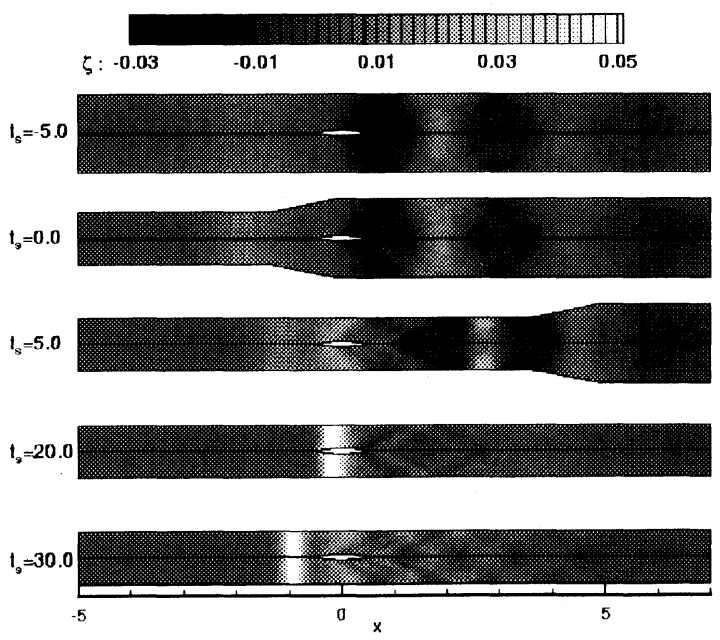

Fig. 18 Evolution of computed wave patterns ( $F n=0.4025$, $\mathrm{B}=1.5 \mathrm{~L} \sim 1.0 \mathrm{~L}, \mathrm{~h}=0.2 \mathrm{~L} \sim 0.1 \mathrm{~L})$

\section{Conclusions}

The numerical scheme introducing the effect of moving grid and CIP method is developed to predict ship waves in the waterway whose depth and breadth vary in longitudinal direction.

Comparison with the results computed by CIP method and MAC method shows the advantage of CIP method to predict accurate ship wave phenomena without expensive computational effort.

The numerical method is applied to simulate ship wave in restricted waterway. It is found that the changes of water depth have an effect on the ship waves but the characteristics of ship waves are little affected by changes of waterway breadth. The ship wave characteristics depend on the breadth of waterway. It can be said that the present study is fundamental research to understand the effect of seabed geometry on ship waves.

\section{References}

1) Kofoed-Hansen, H. and Mikkelsen, A. C. : Wake Wash Form Fast Ferries in Denmark, FAST97, pp. 471-478, 1997.

2) http://www.xontech.com/wdc/public_htm1/ceros/Environm ental lssues with Wake.htm

3) Stumb, S., Fox, K., Dvorak, F. and Elliot, L. : The Prediction, Measurement, and Analysis of Wake Wash from Marine Vessels, Marine Technology, Vol. 36. No. 4, pp248-260, 1999.

4) Kirkegaard, J., Kofoed-Hansen, H. and Elfrink, B. : Wake Wash of High-Speed Craft in Coastal areas, Proc. 26th International Conference on Coastal Eng., pp325-337, 1998.

5) Ertekin, R. C. : Soliton Generation by Moving Disturbance in Shallow Water : Theory, Computation and Experiment, $\mathrm{Ph}$. D. Thesis, University of California, Berkeley, 1984.

6) Chen, X. N. and Sharma, S. D. : A Slender Ship Moving at a Near-Critical Speed in a Shallow Water, J. Fluid Mech., Vol. 291, pp263-285, 1995.

7) Madsen, P. A. and Sorensen, O. R. :A new form of the Boussinesq equations with improved linear dispersion characteristics, Costal Engineering, 18. pp 183-204, 1992.

8) Yabe, T. and Xiao, F. , "Capturing Free Surface and Universal Treatment of Solid, Liquid, Gas by CIP Method", Proc. of CFD Symposium for Free Surface Flows, pp 119-127, 1996.

9) Hung, C. : Extrapolation of Velocity for Inviscid Solid Boundary Condition, AIAA J., Vol. , No. 11, 1987

10) Welch, J. E., Harlow, F. H., Shannin, J. P. and Daly, B. J. : The MAC Method, Los Alamos Scientific Lab., Report, LA-3425, Los Alamos, N.W., 1966.

11) Cooperative Experiments on Wigley Parabolic Models in Japan, $17^{\text {th }}$ ITTC R.C. report, 1983. 